Published in final edited form as:

Pediatr Rev. 2018 November ; 39(11): 533-541. doi:10.1542/pir.2017-0285.

\title{
Campylobacter Infections in Children
}

\author{
Rebecca G. Same, MDPranita D. Tamma, MD, MHS ${ }^{\star}$ \\ *Division of Pediatric Infectious Diseases, Department of Pediatrics, The Johns Hopkins \\ University School of Medicine, Baltimore, MD
}

\section{Abstract}

Education Gap-Campylobacter is one of the 2 most common causes of foodborne illness in the United States. It most commonly occurs in children younger than 5 years of age.

Campylobacter species can cause a wide range of syndromes, from asymptomatic infections to severe systemic infections.

Objectives-After completing this article, readers should be able to:

1. Recognize that Campylobacter is a common cause of foodborne illness in the United States and internationally.

2. Understand the indications for testing and the treatment of Campylobacter infection.

\section{INTRODUCTION}

Campylobacter species are an important cause of infection throughout the world, especially in young children. Campylobacter jejuni is the most common Campylobacter species in the United States, where it is 1 of the top 2 causes of foodborne illness. (1) Other important species include Campylobacter upsaliensis, Campylobacter lari, and Campylobacter fetus, which can cause serious systemic infections in all age groups, particularly in immunocompromised patients.

Campylobacter species infections cause gastroenteritis and typically present with diarrhea that may or may not be bloody, emesis, and abdominal pain. Campylobacter infection in children can mimic intussusception, appendicitis, or inflammatory bowel disease (IBD). Immune-mediated complications of Campylobacter infection include reactive arthritis and Guillain-Barré syndrome (GBS). Campylobacter infection is a nationally notifiable disease, and confirmed cases should be reported through the Centers for Disease Control and Prevention (CDC) Foodborne Diseases Active Surveillance Network.

Most Campylobacter infections are mild and self-limited and require only supportive care, although some may lead to severe dehydration. Serious infection or infections in immunocompromised hosts may benefit from treatment with macrolide antibiotics.

AUTHOR DISCLOSURE Dr Same has disclosed no financial relationships relevant to this article. Dr Tamma has disclosed that she has an investigator-initiated research grant from Merck unrelated to this article. This commentary does not contain a discussion of an unapproved/investigative use of a commercial product/device. 
Increasing rates of fluoroquinolone resistance have been observed in Campylobacter isolates. (2)

The largest source of Campylobacter infections is animals, especially wild and domestic birds. Strategies to prevent Campylobacter infection include thoroughly cooking poultry, preventing cross-contamination with other foods, properly chlorinating water and pasteurizing milk, and practicing hand hygiene after interacting with animals.

\section{MICROBIOLOGY}

The Campylobacter genus is composed of gram-negative, motile, curved or spiral-shaped bacilli. The name Campylobacter is derived from Greek and Latin and means "curved rod." As of 2014, the genus included 26 species, approximately half of which cause disease in humans. (3) The organisms that are most commonly associated with infection in humans are C jejuni and Campylobacter coli. In 2015, 88\% of all isolates that were speciated were $C$ jejuni. (1) $C$ jejuni contains 2 subspecies: $C$ jejuni subspecies jejuni (typically referred to as C jejuni), which is the most commonly isolated cause of Campylobacter infection in the United States, and $C$ jejuni subspecies doylei, which is much rarer. Other Campylobacter species that cause infection in humans are $C$ fetus, $C$ upsaliensis, $C$ lari, and Campylobacter hyointestinalis. This review focuses on $C$ jejuni and $C$ coli, with a brief discussion of other, less common species at the end.

\section{PATHOGENESIS}

There are multiple organisms and host factors that enable Campylobacter infection in humans. Studies in healthy volunteers as well as observational data from outbreaks have demonstrated that the inoculum required to cause Campylobacter enteritis in humans can be as low as 500 organisms. (4) (5) (6) Disruption of the gastric acid barrier allows for pathogenic flora, such as Campylobacter, to survive and flourish. Therefore, patients with reduced gastric acidity, including those receiving proton pump inhibitors, may be at higher risk for infection with Campylobacter. (7)(8)(9)(10) The incubation period is relatively short, ranging from 1 to 7 days, with an average of 3 days. Higher inocula may result in shorter incubation periods. Infection is typically established in the distal ileum and colon and initially causes a noninflammatory diarrhea. This is followed by a locally invasive stage that leads to cell damage and inflammation that can present with dysentery, which is occasionally followed by translocation across the intestinal epithelium, causing lymphadenitis and extraintestinal infection. Histologically, infection is identical to salmonellosis or shigellosis and is marked by acute mucosal inflammation and edema, which can include infiltration of the lamina propria and crypt abscess formation. (11) (12)

The exact mechanisms of infection are not completely understood, but several virulence factors have been identified, including flagella, plasmids, adhesins, and chemotactic factors. (13) Initial infection is established when bacteria attach to intestinal epithelial cells via fimbriaelike filaments. Colonization of the gastrointestinal tract is then facilitated by flagella and chemotactic factors. Campylobacter flagellins do not seem to provoke proinflammatory cytokines, which may allow Campylobacter to evade innate immune response, 
differentiating it from other intestinal pathogens, including Salmonella. (14) There are a variety of other surface proteins and adhesins that facilitate colonization and invasion of intestinal epithelial cells. Some isolates of Campylobacter contain a high-molecular-weight plasmid, pVir, that has been associated with bloody stools and is thought to contribute to invasiveness. (15)

The immune response to Campylobacter infection seems to be primarily humoral. Campylobacter-specific serum immunoglobulin (Ig) A level rises rapidly during the first 2 weeks after infection and then declines slowly over the next month. The IgM and IgG antibody levels rise more slowly and peak 2 to 3 weeks after development of symptoms. (16) Prolonged and severe infection has been reported in patients with hypogammaglobulinemia. (17) The role of cellular immunity in Campylobacter infection is less well defined, but it does seem to be important because patients with human immunodeficiency virus (HIV) are also at risk for more severe and prolonged disease as well as higher rates of extraintestinal infection. (18) In immunocompetent patients, infection with Campylobacter provides some immunity against future infection, especially infection with the same strain.

\section{EPIDEMIOLOGY}

Campylobacter is a leading cause of acute diarrhea domestically and internationally. In the United States, Campylobacter and Salmonella are the 2 most common causes of foodbome illness. In 2016 the incidence of Campylobacter enteritis confirmed by culture or cultureindependent diagnostic tests (CIDTs) was 17.4 infections per 100,000 persons, making it the most commonly identified cause of foodbome illness in the United States. Campylobacter has been tracked through the Foodbome Diseases Active Surveillance Network since 1996 and has been a notifiable disease since 2015. Most Campylobacter infections are mild and self-limited, but in 2016, 20\% of reported infections resulted in hospitalization and 26 attributable deaths were identified (mortality, 0.3\%). (19)

In the United States, the incidence of infection is highest in children younger than 5 years of age, but there is also a secondary peak in young adults. Infection rates are highest in the summer, and most infections are domestically acquired, although Campylobacter enteritis is also seen in returning international travelers, (1) In developing countries, the infection is hyperendemic, and symptomatic infection occurs almost exclusively in infants and young children, who can be infected repeatedly. Subsequent infections tend to be asymptomatic, making symptomatic disease rare in older children or adults. (20) (21)

Campylobacter enteritis is usually sporadic; only $0.4 \%$ of identified infections in 2015 were associated with an outbreak. (1) Transmission to humans occurs through ingestion of contaminated food or water or by direct contact with fecal material from infected people or animals. Animal reservoirs, induding wild and domestic birds and other animals, are the most common source of Campylobacter infections. Multiple studies have shown that most broiler chicken flocks, $60 \%$ to $80 \%$, may be contaminated with Campylobacter at slaughter. (22) The main vehicle of transmission to humans is improperly cooked poultry; 1 study suggested that $48 \%$ of Campylobacter infections are attributable to poultry exposure. (23) Direct acquisition of infection from animals is less common and is usually associated with 
occupational exposure to poultry. Infection has been reported in association with domestic pets, especially puppies and kittens, that have Campylobacter diarrhea. (23) In the fall of 2017 an outbreak of multidrug-resistant Campylobacter was linked to puppies sold through a national pet store chain. (24) Person-to-person transmission is rarer, but caretakers of children or other individuals in diapers are at increased risk. Sexual transmission has also been reported, especially in men who have sex with men. (25)

Outbreaks have been reported in association with the distribution of contaminated water or milk. Campylobacter can survive in water or milk for many weeks at low temperatures. Because Campylobacter is nearly ubiquitous in the environment, decontamination is the most important strategy to prevent transmission. Waterborne outbreaks have occurred in association with unchlorinated water or failed chlorination systems, and milk-associated outbreaks have been connected to raw milk or failures in the pasteurization process. (26) (27) (28) (29) Importantly, milk-borne outbreaks have been associated with raw milk that had passed routine testing because $C$ jejuni can sometimes be difficult to culture. (29)

\section{CLINICAL PRESENTATION}

The most common illness caused by $C$ jejuni and $C$ coli is gastroenteritis. Children present with diarrhea and abdominal pain, often accompanied by vomiting and fever. Infection can result in significant dehydration. Bloody stools are also seen in at least half of infected children. (30)(31) Fevers can be prominent, and seizures, meningismus, and encephalopathy have all been reported in association with Campylobacter infection. (26)(32) Bacteremia is rare and is seen primarily in patients who are immimocompromised. (33)(18)

Perinatal $C$ jejuni infection is rare but has been described. It can result in abortion, premature labor, and/or neonatal septicemia and meningitis. Symptomatic gastroenteritis in neonates is also possible, which may present with only grossly bloody stools or fever. Infection is generally acquired perinatally from mothers that shed Campylobacter either symptomatically or asymptomatically. Outbreaks in neonatal nurseries due to nosocomial spread have been reported. (34)

Campylobacter infection can also mimic other gastrointestinal illnesses. Infants with Campylobacter enteritis may present with only bloody stools and vomiting without fever, which can be mistaken for intussusception. In older children, acute Campylobacter ileocecitis can cause severe right lower quadrant pain without diarrhea mimicking appendicitis. (35)(36) Campylobacter enteritis most commonly progresses from the small bowel distally, but rarely patients with severe infection may present with only colitis and bloody diarrhea that can be confused with IBD. (37) Imaging can be helpful in excluding other intra-abdominal processes, such as intussusception. The acute inflammatory changes of Campylobacter infection observed on histologic analysis should help differentiate it from the chronic changes of IBD. It has also been suggested that Campylobacter infection may play a role in the development of IBD. (38)

Although the manifestations can be variable, Campylobacter gastroenteritis is usually relatively mild and self-limited, with symptoms lasting 24 to 48 hours in most patients. 
Excretion of the organism can last much longer than clinical symptoms. On average, shedding lasts 2 to 3 weeks, but it can range from 3 days to several months. (31) Shedding tends to be prolonged in younger children and patients with immunodeficiencies. Rarely, symptoms may persist for several weeks or relapse may occur after initial resolution. Chronic or recurrent infection is more common in immunocompromised patients, who can develop a prolonged relapsing syndrome that mimics IBD. This has been described in particular in patients with HIV, in whom long-term carriage and recurrent enteritis can be associated with bacteremia, fever, and debilitating illness. (18)(33)(39)(40)(41) The incidence of Campylobacter infection remains higher in patients with HIV than in the general population, but it has decreased significantly since the introduction of highly active antiretroviral therapy. (41)

\section{COMPLICATIONS}

\section{Early Onset}

Extraintestmal manifestations without enteritis are rare, but septic arthritis, bursitis, osteitis, and soft tissue infections have been reported. Acute extraintestinal complications of enteritis include cholecystitis, peritonitis in patients with peritoneal dialysis, septic pseudoaneurysm, pericarditis, and myocarditis. Erythema nodosum, glomerulonephritis, hemolytic anemia, IgA nephropathy, postinfectious irritable bowel syndrome, and intestinal perforation have all been de-scribed as well.

\section{Late Onset}

Late-onset immune-mediated complications of Campylobacter infection, including arthritis and GBS, have been well described.

Reactive arthritis after Campylobacter enteritis can occur 3 to 40 days (mean, 11 days) after the onset of diarrhea. It is usually oligoarticular and asymmetrical, and it predominantly affects the knees. It is more common and possibly more severe in patients with HLA-B27 phenotype. The development of arthritis does not seem to be related to the severity of the initial illness. Symptoms generally last for up to 21 days, and most patients have spontaneous recovery within 6 months without long-term sequelae. The arthritis is reactive, not infectious; synovial fluid is sterile. Arthritis occurs in up to 7\% of patients, but as many as $20 \%$ of patients report arthralgia. (42)

Both serologic studies and culture surveys suggest that Campylobacter infection is the most commonly identified cause of GBS. Thirty percent to $40 \%$ of cases of GBS develop in patients who were infected with Campylobacter 10 to 14 days earlier. In the United States, 1 in 1,000 patients with Campylobacter enteritis goes on to develop GBS. Some studies have suggested that GBS associated with Campylobacter infection may have a poorer prognosis than GBS associated with other etiologies. (43)

The primary mechanism for Campylobacter-associated GBS is molecular mimicry. The $C$ jejuni lipopolysaccharide resembles GMi ganglioside on peripheral nerve myelin. Infection can lead to the development of cross-reacting antibodies that cause nerve damage. The Miller Fisher variant of GBS, with more prominent cranial nerve involvement resulting in 
ophthalmoplegia, areflexia, and ataxia, is also more common in patients with Campylobacter infection. It is thought to be caused by antibodies that cross-react with Campylobacter lipopolysaccharide and ganglioside GQib in cranial nerve myelin. (44) Certain Campylobacter serotypes, especially Penner $\mathrm{O}_{19}$ and $\mathrm{O}_{41}$, seem to be more commonly associated with GBS. (43) There may be factors other than molecular mimicry that also play a role in the development of Campylobacter-associated GBS. Additional host factors seem to be important; some patients develop antibodies to GMi ganglioside but do not develop neurologic symptoms. There may be an association between HLA types and the development of Campylobacter-associated GBS. (45)

\section{DIAGNOSIS}

Campylobacter enteritis is often clinically indistinguishable from other viral or bacterial gastroenteridites. Diagnostic testing is not always indicated for children who present with acute diarrheal illnesses, with or without fever or vomiting, because determining the cause often does not change clinical management. However, Infectious Diseases Society of America guidelines suggest testing patients with fever or bloody diarrhea or others in whom treatment may be indicated, including anyone with immunodeficiencies. In addition, organism-specific diagnosis can be valuable for the management of outbreaks. Blood cultures are recommended in infants younger than 3 months of age, those with signs of sepsis or systemic manifestations, and immunocompromised patients. (46)

Stool culture is the gold standard for the identification of Campylobacter species. Most laboratories specifically look for Campylobacter in standard stool cultures, but it can be difficult to isolate. Campylobacter grows best on media containing selective antibiotics and in microaerobic conditions with $5 \%$ to $10 \%$ oxygen, $1 \%$ to $10 \%$ carbon dioxide, and some hydrogen. $C$ jejuni and $C$ coli grow best at $107.6^{\circ} \mathrm{F}\left(42^{\circ} \mathrm{C}\right)$. Campylobacter is identified by its characteristic appearance as a comma- or spiral-shaped Gram-negative bacillus as well as oxidase and catalase production. Species-level identification is not typically performed, and differentiation of $C$ jejuni from $C$ coli is not usually necessary for management. Speciation and strain typing can be helpful for epidemiologic purposes or when species other than $C$ jejuni or $C$ coli are suspected. This typing is typically performed at reference laboratories.

Use of CIDTs, including nucleic acid amplification tests, is increasing. (19) These tests are generally more sensitive and have faster turnaround times than traditional culture-based diagnostics. Reverse transcriptase polymerase chain reaction identifies Campylobacter from stool $20 \%$ to $40 \%$ more frequently than culture-based methods. (47) However, because these tests identify the presence of nucleic acid rather than viable organisms, the clinical significance is not always clear. The identification of multiple pathogens is not uncommon and can be difficult to interpret. In addition, CIDTs cannot be used to identify antibiotic susceptibility patterns. Unlike organisms such as Shigella and Salmonella, cultures of Campylobacter often are not performed automatically when the organism is detected by CIDT. Antibiotic resistance to quinolones and tetracyclines is common in Campylobacter isolates, so if treatment is warranted and there is concern for resistance, cultures can still be beneficial after identification by CIDT. (2) 
Serologic testing can be used to detect recent Campylobacter infection in patients with reactive arthritis or GBS who have negative stool studies. Serologic studies are not helpful in the diagnosis of acute Campylobacter infection.

If Campylobacter infection is suspected or confirmed, it is important to attempt to identify the source of infection, mostly to prevent others from becoming infected. Families should be asked about exposures, including consumption of raw milk or contaminated drinking water, undercooked meats or poultry, contaminated fruits or vegetables, and contact with animals (wild and domesticated fowl, puppies, kittens) or their feces. Travel exposure may also be relevant, although most infections are acquired domestically.

\section{MANAGEMENT}

Campylobacter infection typically results in a mild, self-limited enteritis that requires only supportive care. Adequate rehydration, either orally or intravenously, is the mainstay of acute management. Antimotility agents should be avoided due to prolongation of symptoms. (48) Several trials have suggested that probiotics may decrease diarrhea duration and stool frequency in immunocompetent children with gastroenteritis of any cause, although the effect seems to be greater in viral than bacterial gastroenteritis, and the optimal formulation remains unknown. This benefit also has to be weighed against case reports of bacteremia or fungemia with molecularly matched isolates in critically ill or immunocompromised patients who received probiotics. (49) In countries with a high prevalence of zinc deficiency or malnutrition, zinc supplementation reduces the duration of diarrhea in children 6 months to 5 years of age with acute diarrhea. (50)

A meta-analysis of 11 randomized controlled trials of antibiotic treatment versus placebo for the treatment of Campylobacter infections in children and adults showed that antibiotic treatment reduced the duration of symptoms by 1.3 days and the duration of Campylobacter excretion in stool. However, given the self-limited nature of most Campylobacter infections, the relatively modest benefit to treatment with antibiotics, and the risk of development of antibiotic resistance, treatment of un-complicated infection is usually not recommended. (51) Treatment is recommended in patients with severe disease, which includes those with bloody stools, high fever, extraintestinal infection, worsening or relapsing symptoms, or prolonged symptoms that exceed 1 week. It is also recommended for patients with uncomplicated infection who are elderly, pregnant, or immunocompromised due to their risk of severe disease. Both GBS and other late-onset complications of Campylobacter infections are immune-mediated and usually develop once symptoms of acute infection have resolved. Therefore, antibiotics are not routinely included in the management of these Campylobacterassociated syndromes unless patients otherwise meet the criteria for treatment with antibiotics, including prolonged or severe symptomatic infection or immunocompromise.

In patients with uncomplicated infection who merit antibiotic therapy, the recommended treatment is azithromycin $10 \mathrm{mg} / \mathrm{kg}$ per day for 3 days or erythromycin $40 \mathrm{mg} / \mathrm{kg}$ per day in 4 divided doses for 5 days. Treatment usually eradicates Campylobacter from the stool within 2 to 3 days. (52) A longer course (7-14 days) may be necessary in complicated infections or in patients who are immunocompromised. Carbapenems are appropriate 
empirical treatment in patients who are severely ill and unable to tolerate oral treatment, although it is important to complete susceptibility testing to verify carbapenem activity. Treatment for bacteremia should be tailored to available susceptibility testing. Although $C$ jejuni and $C$ coli are usually sensitive in vitro to clindamycin, tetracyclines, and chloramphenicol, data are lacking to support their use dinically. Campylobacter species are inherently resistant to trimethoprim and $\beta$-lactam antibiotics, including penicillin and cephalosporins, so these should be avoided.

\section{Resistance}

Fluoroquinolones may also be effective treatment for Campylobacter infection, but resistance is increasingly common, limiting the effectiveness of these agents for this indication. In the past, this resistance was thought to be, in part, attributable to quinolone use in poultry feeds, but this practice has been banned in the United States since 2005. United States data from 2014 indicated that ciprofloxacin resistance was present in $27 \%$ of $C$ jejuni isolates and $36 \%$ of $C$ coli. (2) Resistance is significantly higher outside the United States and is important to consider in travelers. (53) Fortunately, macrolide resistance remains rare in the United States.

\section{PREVENTION}

Prevention of Campylobacter infection should focus on transmission from animals, milk or waterborne disease, and person-to-person transmission. Contaminated poultry is the most common source of Campylobacter infection, so thoroughly cooking poultry and avoiding cross-contamination with other foods should be emphasized. Campylobacter is killed by heat, so all meat, but especially chicken, should be cooked to proper temperature. Hand hygiene and thorough cleansing of cutting boards and utensils after contact with raw poultry as well as avoiding contact between raw poultry and other foods are also important. Hand hygiene is also important after contact with the feces of dogs and cats, especially puppies and kittens, where high rates of Campylobacter carriage are present. Unpasteurized milk should be avoided, and water should be chlorinated.

People with diarrhea should be excluded from food handling and the care of patients in hospitals and child care centers. They can resume work if infected but asymptomatic, as long as proper hand hygiene is practiced.

Infants and children in diapers who are found to have Campylobacter enteritis should be excluded from child care centers until symptoms have resolved. Treatment of these children with azithromycin or erythromycin can be considered to limit spread. Contact precautions should be used for children who are hospitalized with symptomatic Campylobacter enteritis. (52) Many institutions use syndromic contact isolation for any patient with diarrhea, regardless of the cause.

Previous infection with Campylobacter decreases the risk of future symptomatic infection but is not completely protective, so patients with previous infections should still use the prevention measures detailed previously herein. 


\section{OTHER CAMPYLOBACTER SPECIES}

\section{Campylobacter upsaliensis}

Similar to $C$ jejuni and $C$ coli, $C$ upsaliensis usually causes gastroenteritis, which may present as bloody diarrhea in $25 \%$ of patients, and occasionally bacteremia. It can also occasionally cause vomiting but is usually milder than $C$ jejuni infection. (54) Bacteremia is primarily seen in malnourished or immunocompromised patients. (55) It is more common in children than adults. C upsaliensis was initially isolated from dogs, and they are considered to be its primary reservoir. Infection may be associated with dose contact with dogs.

$C$ upsaliensis is catalase negative or catalase weak and has a different antibiotic susceptibility pattern than $C$ jejuni. It is susceptible to antibiotic agents usually used in Campylobacter-selective media and is, therefore, not detected in cultures using these media. (56) Consequently, the true prevalence of $C$ upsaliensis is not dear, but it is the third most common Campylobacter species (after $C$ jejuni and $C$ coli) recovered in foodbome infections in the United States. (1)

Data are limited regarding treatment of $C$ upsaliensis. Similar to $C$ jejuni and $C$ coli, it is usually treated with macrolides, although resistance has been reported in some studies. Some isolates are susceptible to gentamicin, azithromycin, erythromycin, meropenem, cephalosporins, fluoroquinolones, tetracyclines, and aminoglycosides. (57)

\section{Campylobacter lari}

C lari is the fourth most commonly isolated Campylobacter species in foodbome illnesses. It is common in chickens but relatively rare in humans. It accounts for less than $1 \%$ of Campylobacter isolates from humans and is predominantly seen in immunocompromised patients and neonates. (58) It can be grown on conventional Campylobacter-selective agars under similar conditions as $C$ jejuni. Macrolides are generally effective for treating $C$ lari infections.

\section{Campylobacter fetus}

Cfetus is rare but tends to cause more serious illness than $C$ jejuni or $C$ coli It causes bloodstream infections more commonly than gastroenteritis and is more common in patients at the extremes of age. In 2015 only $0.1 \%$ of laboratory-confirmed cases of Campylobacter in the United States were due to $C$ fetus, but studies have shown that it is responsible for $19 \%$ to 53\% of Campylobacter bacteremia. (1)(33)(59) It may not be identified in stool cultures because it grows best at $77.0^{\circ} \mathrm{F}\left(25^{\circ} \mathrm{C}\right)$ and $98.6^{\circ} \mathrm{F}\left(37^{\circ} \mathrm{C}\right)$ rather than at io $7.6^{\circ} \mathrm{F}$ $\left(42^{\circ} \mathrm{C}\right)$ like Cjejuni. (60)

Three syndromes associated with $C$ fetus bloodstream infection have been described: first, isolated bloodstream infection that is self-limited or rapidly responds to antibiotic therapy; second and most commonly, bloodstream infection assodated with a focal infection such as meningitis, pneumonia, endocarditis, or thrombophlebitis; third, chronic relapsing/remitting bacteremia, seen most commonly in immunocompromised patients. Perinatal infection has also been described and can be assodated with spontaneous abortion, preterm labor, and 
neonatal septicemia or meningitis. Perinatal infection with $C$ fetus is rare but tends to be more severe than with $C$ jejuni.

Although $C$ fetus is often susceptible to ampidllin and gentamicin, carbapenems are preferred first-line agents pending susceptibility testing. $C$ fetus is typically susceptible to ampicillin, extended-spectrum cephalosporins, meropenem, imipenem, aminoglycosides, and erythromycin. (61)

\section{AUTHOR DISCLOSURE}

Dr Same has disclosed no financial relationships relevant to this article. Dr Tamma has disclosed that she has an investigator-initiated research grant from Merck unrelated to this article. This commentary does not contain a discussion of an unapproved/investigative use of a commercial product/device.

\section{ABBREVIATIONS}

$\begin{array}{ll}\text { CDC } & \text { Centers for Disease Control and Prevention } \\ \text { CIDT } & \text { culture-independent diagnostic testing } \\ \text { GBS } & \text { Guillain-Barré syndrome } \\ \text { HIV } & \text { human immunodeficiency virus } \\ \text { IBD } & \text { inflammatory bowel disease } \\ \text { Ig } & \text { immunoglobulin }\end{array}$

\section{References}

1. Centers for Disease Control and Prevention. Foodbome Diseases Active Surveillance Network (FoodNet): FoodNet 2015 Surveillance Report (Final Data). Atlanta, GA: US Department of Health and Human Services, CDC; 2017.

2. Centers for Disease Control and Prevention. National Antimicrobial Resistance Monitoring System for Enteric Bacteria (NARMS): Human Isolates Surveillance Report for 2014 (Final Report). Atlanta, GA: US Department of Health and Human Services, CDC; 2016.

3. Campylobacter Fitzgerald C.. Oin Lab Med. 2015;35(2):289-298

4. Black RE, Levine MM, Clements ML, Hughes TP, Blaser MJ. Experimental Campylobacter jejuni infection in humans. J Infect Dis. 1988;157(3):472-479 [PubMed: 3343522]

5. Hara-Kudo Y, Takatori K. Contamination level and ingestion dose of foodbome pathogens associated with infections. Epidemiol Infect. 2011;139(10):1505-1510 [PubMed: 21205441]

6. Robinson DA. Infective dose of Campylobacter jejuni in milk. Br Med J (clin Res Ed). 1981;282(6276):1584

7. Neal KR, Scott HM, Slack RC, Logan RF. Omeprazole as a risk factor for Campylobacter gastroenteritis: case-control study. BMJ. 1996:312 (7028)414-415 [PubMed: 8601113]

8. Hassing RJ, Verbon A, de Vìsser H, Hofman A, Strieker BH. Proton pump inhibitors and gastroenteritis. Eur J Epidemiol. 2016:31(10):1057-1063 [PubMed: 26960438]

9. Brophy S, Jones KH, Rahman MA, et al. Incidence of Campylobacter and Salmoneüa infections following first prescription for PPI:a cohort study using routine data. Am J Gastroenterol 2013:108(7):1094-1100 [PubMed: 23588238]

10. Bavishi C, Dupont HL. Systematic review: the use of proton pump inhibitors and increased susceptibility to enteric infection. Aliment Pharmacol Ther. 2011;34(11-12):1269-1281 [PubMed: 21999643] 
11. van Spreeuwel JP, Duursma GC, Meijer CJ, Bax R, Rosekrans PC, Lindeman J. Campylobacter colitis: histological immunohistochemical and ultrastructural findings. Gut. 1985:26(9):945-951 [PubMed: 4029720]

12. Mee AS, Shield M, Burke M. Campylobacter colitis: differentiation from acute inflammatory bowel disease. J R Soc Med. 1985;78(3):217-223 [PubMed: 3973886]

13. Bolton DJ. Campylobacter virulence and survival factors. Food Microbiol. 2015;48:99-108 [PubMed: 25790997]

14. de Zoete MR, Keestra AM, Wagenaar JA, van Putten JP. Reconstitution of a functional Toll-like receptor 5 binding site in Campylobacter jejuni flagellin. J Biol Chem. 2010:285(16):1214912158 [PubMed: 20164175]

15. Tracz DM, Keelan M, Ahmed-Bentley J, Gibreel A, Kowalewska-Grochowska K, Taylor DE. pVir and bloody diarrhea in Campylobacter jejuni enteritis. Emerg Infect Dis. 2005;11(6):838-843 [PubMed: 15963277]

16. Blaser MJ, Duncan DJ. Human serum antibody response to Campylobacter jejuni infection as measured in an enzyme-linked immunosorbent assay. Infect Immun. 1984;44(2):292-298 [PubMed: 6715034]

17. Melamed I, Bujanover Y, Igra YS, Schwartz D, Zakuth V, Spirer Z. Campylobacter enteritis in normal and immunodefirient children. Am J Dis Child. 1983:137(8)752-753 [PubMed: 6869333]

18. Tee W, Mijch A. Campylobacter jejuni bacteremia in human immunodeficiency virus (HIV)infected and non-HIV-infected patients: comparison of clinical features and review. Clin Inject Dis. 1998;26(1):91-96

19. Marder EP, Cieslak PR, Cronquist AB, et al. Incidence and trends of infection with pathogens transmitted commonly through food and the effect of increasing use of culture-independent diagnostic tests on Surveillance-Foodbome Diseases Active Surveillance Network, 10 U.S. sites, 2013-2016. MMWR Morb Mortal Wl·ly Rep. 2017:66 (15);397-403

20. Calva JJ, Ruiz-Palados GM, Lopez-Vidal AB, Ramos A, Bojalil R. Cohort study of intestinal infection with Campylobacter in Mexican children. Lancet. 1988;1(8584):503-506 [PubMed: 2893920]

21. Platts-Mills JA, Kosek M. Update on the burden of Campylobacter in developing countries. Curr Opin Infict Dis. 2014;27(5):444-450

22. Hermans D, Pasmans F, Messens W, et al. Poultry as a host for the zoonotic pathogen Campylobacter jejuni. Vector Borne Zoonotic Dis. 2012;12(2):89-98 [PubMed: 22133236]

23. Saeed AM, Harris NV, DiGiacomo RF. The role of exposure to animals in the etiology of Campylobacter jejuni/coli enteritis. Am J Epidemiol. 1993;137(1):108-114 [PubMed: 8434569]

24. Centers for Disease Control and Prevention (CDC). Multistate outbreak of multidrug-resistant Campylobacter infections linked to contact with pet store puppies. Available at: https:// www.cdc.gov/campylobacter/outbreaks/puppies-9-i7/index.html. Published October 3, 2017. Updated 2017. Accessed October 27, 2017.

25. Gaudreau C, Rodrigues-Coutlée S, Pilon PA, Coutlée F, Bekal S. Long-lasting outbreak of erythromycin- and ciprofloxacin-resistant Campylobacter jejuni subspecies jejuni from 2003 to 2013 in men who have sex with men, Quebec, Canada. Clin Infect Dis. 2015;61(1O):1549-I552 [PubMed: 26187024]

26. Jones PH, Willis AT, Robinson DA, Skirrow MB, Josephs DS. Campylobacter enteritis associated with the consumption of free school milk. J Hyg (Lond). 1981;87(2):155-162 [PubMed: 6895230]

27. Vogt RL, Sours HE, Barrett T, Feldman RA, Dickinson RJ, Witherell L. Campylobacter enteritis associated with contaminated water. Ann Intern Med. 1982;96(3):292-296 [PubMed: 7059090]

28. Wood RC, MacDonald KL, Osterholm MT. Campylobacter enteritis outbreaks associated with drinking raw milk during youth activities: aio-yearreviewofoutbreaksinthe United States. JAMA. 1992:268(22):3228-3230 [PubMed: 1433764]

29. Davis KR, Dunn AC, Burnett C, et al. Campylobacter jejuni infections associated with raw milk consumption-Utah, 2014. MMWR Morb Mortal Wkly Rep. 2016;65(12):301-305 [PubMed: 27031585]

30. Blaser MJ, Berkowitz ID, LaForce FM, Cravens J, Reller LB, Wang WL. Campylobacter enteritis: clinical and epidemiologic features. Ann Intern Med. 1979;91(2):179-185 [PubMed: 380433] 
31. Karmali MA, Fleming PC. Campylobacter enteritis in children. J Pediatr. 1979;94(4):527-533 [PubMed: 430287]

32. Levy I, Weissman Y, Sivan Y, Ben-Ari J, Scheinfeld T. Acute encephalopathy associated with Campylobacter enteritis. Br Med] (Clin Res Ed). 1986;293(6544):424

33. Femández-Cruz A, Muñoz P, Mohedano R, et al. Campylobacter bacteremia: clinical characteristics, incidence, and outcome over 23 years. Medicine (Baltimore). 2010;89(5):319-330 [PubMed: 20827109]

34. Goossens H, Henocque G, Kremp L, et al. Nosocomial outbreak of Campylobacter jejuni meningitis in newborn infants. Lancet. 1986;2(8499);146-149 [PubMed: 2873408]

35. Puylaert JB, Vermeijden RJ, van der Werf SD, Doombos L, Koumans RK. Incidence and sonographic diagnosis of bacterial ileocaecitis masquerading as appendicitis. Lancet. 1989:2(8654):84-86 [PubMed: 2567879]

36. Puylaert JB, Van der Zant FM, Mutsaers JA. Infectious ileocedtis caused by Yersinia, Campylobacter, and Salmonella: clinical, radiological and US findings. Eur Radiol. 1997;7(1):3-9 [PubMed: 9000386]

37. Loss RW Jr, Mangla JC, Pereira M. Campylobacter colitis presenting as inflammatory bowel disease with segmental colonic ulcerations. Gastroenterology. 1980;79(1):138-140 [PubMed: 7380209]

38. Castaño-Rodríguez N, Kaakoush NO, Lee WS, Mitchell HM. Dual role of Helicobacter and Campylobacter species in IBD: a systematic review and meta-analysis. Gut. 2017;66(2):235-249 [PubMed: 26508508]

39. Sorvillo FJ, Lieb LE, Waterman SH. Incidence of campylobacteriosis among patients with AIDS in Los Angeles County. J Acquir Immune Defic Syndr. 1991;4(6):598-602 [PubMed: 2023099]

40. Ruiz-Contreras J, Ramos JT, Hemández-Sampelayo T, de José M, Clemente J, Gurbindo MD; The Madrid HIV Pediatric Infection Collaborative Study Group. Campylobacter sepsis in human immunodeficiency virus-infected children. Pediatr Inject Dis J. 1997;16(2):251-253

41. Larsen IK, Gradel KO, Helms M, et al. Non-typhoidal Salmonella and Campylobacter infections among HIV-positive patients in Denmark. Scand J Infect Dis. 2011;43(1):3-7 [PubMed: 20849366]

42. Pope JE, Krizova A, Garg AX, Thiessen-Philbrook H, Ouimet JM. Campylobacter reactive arthritis: a systematic review. Semin Arthritis Rheum. 2007;37(1):48-55 [PubMed: 17360026]

43. Nachamkin I, Alios BM, Ho T. Campylobacter species and Guillain-Barré syndrome. Clin Microbiol Rev. 1998;11(3):555-567 [PubMed: 9665983]

44. Jacobs BC, Hazenberg MP, van Doom PA, Endtz HP, van der Meché FG. Cross-reactive antibodies against gangliosides and Campylobacter jejuni lipopolysaccharides in patients with Guillain-Barré or Miller Fisher syndrome. J Infect Dis. 1997;175(3)729-733 [PubMed: 9041356]

45. Koga M, Yuki. N, Kashiwase K, Tadokoro K, Juji T, Hirata K. Guillain-Barré and Fisher's syndromes subsequent to Campylobacter jejuni enteritis are associated with HLA-B54 and Cwi independent of anti-ganglioside antibodies. J Neuroimmunol. 1998;88(1-2):62-66 [PubMed: 9688325]

46. Shane A, Mody R, Crump J, et al. 2017 Infectious Diseases Society of America clinical practice guidelines for the diagnosis and management of infectious diarrhea. Clin Inject Dis. 2017;65(12): 1963-1973

47. Bonilauri P, Bardasi L, Leonelli R, et al. Detection of food hazards in foods: comparison of real time polymerase chain reaction and cultural methods. Ital J Food Saj 2016;5(1):5641

48. Smith GS, Blaser MJ. Fatalities associated with Campylobacter jejuni infections. JAMA. 1985;253(19):2873-2875 [PubMed: 3989964]

49. Freedman SB, Ali S, Oleszczuk M, Gouin S, Hartling L. Treatment of acute gastroenteritis in children: an overview of systematic reviews of interventions commonly used in developed countries. Evid Based Child Health. 2013;8(4):1123-1137 [PubMed: 23877938]

50. Lazzerini M, Wanzira H. Oral zinc for treating diarrhoea in children. Cochrane Database Syst Rev. 2016;12:CD005436 [PubMed: 27996088] 
51. Temhag A, Asikainen T, Giesecke J, Ekdahl K. A meta-analysis on the effects of antibiotic treatment on duration of symptoms caused by infection with Campylobacter species. Clin Infect Dis. 2007:44(5):696-700 [PubMed: 17278062]

52. American Academy of Pediatrics. Campylobacterinfections In: Kimberlin D, Brady M, Jackson M, Long S, eds. Red Book: 2015 Report of the Committee on Infectious Diseases. 30th ed. Elk Grove Village, IL: American Academy of Pediatrics; 2015:273

53. Campylobacter Sentinel Surveillance Scheme Collaborators. Ciprofloxacin resistance in Campylobacter jejuni: case-case analysis as a tool for elucidating risks at home and abroad. J Antimicrob Chemother. 2002;50(4):561-568 [PubMed: 12356802]

54. Jimenez SG, Heine RG, Ward PB, Robins-Browne RM. Campylobacter upsaliensis gastroenteritis in childhood. Pediatr Inject Dis J. 1999;18(11):988-992

55. Lastovica AJ, Le Roux E, Penner JL. "Campylobacter upsaliensis" isolated from blood cultures of pediatric patients. J Clin Microbiol. 1989;27(4):657-659 [PubMed: 2723034]

56. Goossens H, Pot B, Vlaes L, et al. Characterization and description of "Campylobacter upsaliensis" isolated from human feces. J Clin Microbiol. 1990;28(5):1039-1046 [PubMed: 2351720]

57. Vandenberg O, Houf K, Douat N, et al. Antimicrobial susceptibility of clinical isolates of nonjejuni/coli campylobacters and arcobacters from Belgium. J Antimicrob Chemother. 2006;57(5): 908-913 [PubMed: 16533825]

58. Chiu CH, Kuo CY, Ou JT. Chronic diarrhea and bacteremia caused by Campylobacter lari in a neonate. Clin Inject Dis. 1995:21 (3)700-701

59. Pacanowski J, Lalande V, Lacombe K, et al.; CAMPYL Study Group. Campylobacter bacteremia: clinical features and factors associated with fatal outcome. Clin Inject Dis. 2008;47(6)790-796

60. Wagenaar JA, van Bergen MA, Blaser MJ, Tauxe RV, Newell DG, van Putten JP. Campylobacter jetus infections in humans: exposure and disease. Clin Inject Dis. 2014;58(11):1579-1586

61. Tremblay C, Gaudreau C, Lorange M. Epidemiology and antimicrobial susceptibilities of hi Campylobacter fetus subsp. jetus strains isolated in Québec, Canada, from 1983 to 2000. J Clin Microbiol. 2003;41(1):463-466 [PubMed: 12517895] 


\section{Summary}

- $\quad$ On the basis of strong evidence, Campylobacter is a common cause of acute diarrhea throughout the world, and Campylobacter jejuni is the most commonly isolated Campylobacter species. (1)

- $\quad$ On the basis of strong evidence, most Campylobacter infections result in a mild, self-limited enteritis, but Campylobacter can also cause invasive disease, including bacteremia. Campylobacter is also the most commonly identified cause of Guillain-Barré syndrome and can cause reactive arthritis.

- $\quad$ Based on strong clinical evidence, stool culture remains the gold standard for the diagnosis of Campylobacter infection, but culture-independent diagnostic testing is also sensitive. On the basis of consensus, species-level identification of Campylobacter isolates is usually not necessary for clinical management.

- Campylobacter is a nationally notifiable disease. Any confirmed cases should be reported through the Centers for Disease Control and Prevention Foodborne Diseases Active Surveillance Network.

- $\quad$ Based on strong evidence, antibiotic treatment of Campylobacter infections results in a modest reduction of duration of infection (1 day) and a reduced duration of shedding. However, based on consensus, treatment is not recommended for uncomplicated infection in immunocompetent hosts due to the mild nature of infection, modest benefit, and risk of development of antibiotic resistance. Treatment with azithromycin or erythromycin is recommended for severe infections or infections in immunocompromised hosts.

- Based on an abundance of evidence, important practices for the prevention of Campylobacter infection are thoroughly cooking poultry, avoiding crosscontamination between poultry and other foods, chlorination of water, pasteurization of milk, and observation of hand hygiene after contact with animals and their feces. 